\title{
La Gestión por Procesos en las Pymes de Barranquilla: Factor Diferenciador de la Competitividad Organizacional
}

\author{
Karelis del C. Barrios-Hernández ${ }^{1}$, Jheison A. Contreras Salinas ${ }^{2}$ y Enohemit Olivero-Vega ${ }^{1}$ \\ (1) Facultad de Administración y Negocios, Universidad Simón Bolívar, Barranquilla-Colombia. \\ (e-mail: kbarrios22@unisimonbolivar.edu.co; karelisbarrios03@gmail.com) \\ (2) Facultad de Ingeniería, Universidad Simón Bolívar, Barranquilla-Colombia
}

Recibido Jun. 25, 2018; Aceptado Ago. 28, 2018; Versión final Oct. 9, 2018, Publicado Abr. 2019

\begin{abstract}
Resumen
Con el objetivo de valorar como la gestión por procesos influye en la competitividad y organización de las pequeñas y medianas empresas con más de cinco años en actividad y pertenecientes al sector comercio y servicio, se propuso un estudio que considera las tendencias globales que orientan y sugieren mayor dinámica desde los componentes organizacionales. Se planteó una investigación cualitativa apoyada en la revisión documental que resultó valiosa para contrastar los referentes bibliográficos con la actividad del sector real. Los principales hallazgos destacan que la competitividad es un tema que con el pasar de los años mantiene su vigencia en las organizaciones empresariales y está en continua evolución. Como resultado final se encontró que, la gestión por procesos aporta de manera significativa al impulso de la competitividad empresarial, orientándola hacia la coordinación y explotación eficiente de los recursos que se dispone en la organización.
\end{abstract}

Palabras clave: competitividad; gestión; procesos; pymes; mercado

\section{The management by Processes in the SMEs of Barranquilla: Differentiating Factor of the Organizational Competitiveness}

\begin{abstract}
In order to assess how process management influences the competitiveness and organization of small and medium enterprises with more than five years in activity and belonging to the trade and service sector, a study was proposed considering the global trends that guide and suggest greater dynamics from the organizational components. A qualitative research based on the documentary review was proposed, which was valuable to contrast the bibliographic references with the activity of the real sector. The main findings highlight that competitiveness is an issue that with the passing of time maintains its validity in business organizations and is in continuous evolution. As a final result it was found that, the management by processes contributes in a significant way to the impulse of managerial competitiveness, oriented it to the coordination and efficient exploitation of the resources that are available in the organization.
\end{abstract}




\section{INTRODUCCIÓN}

Hacia el año de 1980, Michael Porter traza las primeras teorías relacionadas con competitividad, motivado por la crisis de la industria del petróleo y el ascenso de la industria japonesa como líder económico, que despertaba la inquietud de la industria norteamericana y europea (Smit, 2010). Sus teorías impactan profundamente el mundo empresarial con la publicación de su libro Estrategia Competitiva, en el que establece las cinco fuerzas de la competencia: rivalidad entre empresas existentes, ingreso potencial de otras empresas, amenaza de productos sustitutos, poder de negociación de los clientes y poder de negociación de los proveedores (Benítez, 2012). Según el análisis realizado por García et al. (2014), de diferentes autores con respecto a la competitividad, ésta se traduce en posiciones ventajosas dentro del mercado para garantizar la sostenibilidad de la empresa dentro de éste. Así también, contempla aspectos no comunes que pueda toda organización disponer para lograr resultados de gran impacto en medio de un ámbito donde diversos actores realizan actividades similares (Nechaev et al., 2014). Entre los autores analizados, se pueden condensar varios aspectos para definir la competitividad en términos de capacidad de una empresa de penetrar en el mercado, logar una posición favorable en términos comparativos con sus competidores, de manera sostenible, con habilidades administrativas y manejo adecuado de sus recursos, ofreciendo la mejor propuesta de producto en términos de calidad y precio, generando dividendos a sus accionista y valor a la sociedad. Las empresas compiten entre sí por una porción del mercado y las naciones o entes territoriales compiten por segmentos de mercados globales (Porter y Kramer, 2018).

Porter establece desde 1980 que las empresas tienen estrategias competitivas que deben enfocarse en el entorno del mercado en que desean competir, pues sus opciones estratégicas están limitadas por él, planteando su análisis en aspectos al exterior de la empresa, para a partir de ahí definir la manera en que debe funcionar su estructura empresarial, esta sugerencia se hace esencial pues en la medida que el mercado es contemplado, se puede avistar aquellos componentes que en el corto, mediano o largo plazo pueden impactar de una forma u otra en las organizaciones (Gupta et al., 2016). No obstante, los aspectos internos no son desconocidos pues en conjunto se estiman surtidores para promover compendios que facilitan la atención de las exigencias que el mercado actual va marcando (Graham, 2016). Así las cosas, se puede enunciar que la importancia de la competitividad radica en que es determinante para su éxito o fracaso de las células productivas (Porter y Heppelmann, 2015) pues su finalidad es ubicar a la empresa en una posición sustentable y rentable, basando su estrategia en costos y diferenciación con respecto a la competencia, buscando que el cliente perciba el valor del producto como algo mayor a su costo y por lo tanto esté dispuesto a pagarlo, esto lo que algunos investigadores han alineado con conceptos de valores agregados que ponderan tanto los productos como servicios, logrando impactar al cliente de tal forma que se logra superar la expectativa inicial del mismo (Porter, 2015). Por lo citado, plantearse un análisis de la cadena de valor, como herramienta para lograr llevar a la empresa a diseñar una estrategia basada en diferenciación y en costos para ser más competitiva, es un ejercicio oportuno para toda organización (Yusuf et al., 2014). En esta evaluación de la cadena de valor, se presenta a la empresa como una desagregación de grandes bloques de actividades, de manera que se permite fácilmente identificar aquellas instancias en las que se genera mayor valor para el cliente o consumidor y, por lo tanto, este puede estar dispuesto a pagar su costo. Cada actividad puede ser generadora de valor, ningún eslabón debe desconocerse pues las oportunidades de competencia se pueden derivar de cualquier parte del proceso (Benítez, 2012).

Las pequeñas y medianas empresas (pymes) en Colombia, son un motor económico de grandes proporciones, pues según Confecámaras hacia el año 2016 se incrementó la creación de empresas en un 21.7\% en comparación con el año 2015; en 2016 se instituyeron 299.632 empresas en el país, 76.794 sociedades y 222.838 personas naturales; de las 299.632 unidades económicas creadas en este periodo, la mayor concentración se encuentra en el sector comercio $(37,1 \%)$, seguido de alojamiento y servicios de comida $(13,1 \%)$, industria manufacturera $(10,7 \%)$, actividades profesionales y científicas $(7,6 \%)$, construcción $(5,8 \%)$, actividades de servicios administrativos y de apoyo $(4,4 \%)$ y otras actividades de servicios $(4 \%)$. Por su mayor participación en la estructura de actividad económica, estos siete sectores dan cuenta del 82,6\% del total de unidades empresariales que se originaron en este año (Confecámaras, 2016). El principal impacto de las pymes se ve reflejado en la generación de empleo, por lo cual motivar el incremento de la competitividad de las mismas, es una tarea que involucra tanto a los entes del Estado, como universidad y organizaciones no gubernamentales (Consuegra et al., 2017).

\section{FUNDAMENTACIÓN TEÓRICA}

Con la finalidad de proveer argumentos teóricos que permitan dar una interpretación coherente a los hallazgos del estudio, se desarrolla de forma concreta una revisión de los postulados de Porter (1980), respecto a las ventajas competitivas y la pertinencia que tienen en la actual dinámica económica en que se desenvuelve las pymes; asimismo se reflexiona sobre la necesidad de revisar el enfoque de las estrategias puras planteadas hace más de 30 años por este autor. Por otra parte, la gestión por procesos cuenta con especial atención, por 
ello, se describen algunos elementos teóricos necesarios para comprender cómo pueden estos aportar a la competitividad de la organización.

\section{Competitividad}

La literatura ofrece un amplio desarrollo sobre a lo que competitividad respecta, sin embargo, se tomara como fundamento teórico los postulados de Porter (2015) respecto a la posibilidad de crear ventajas competitivas para lograr un desempeño superior en un sector determinado. Inicialmente, se puede destacar que la visión del autor consiste en definir esta ventaja como la forma en la cual una empresa, país e incluso persona puede colocarse en una posición diferenciadora relativamente superior frente otros con base en factores y elementos propios difíciles de emular; lo que se traduce en una característica única y diferencial (Porter, 2015).

Sin embargo, para alcanzar el desarrollo pleno de una ventaja competitiva en el sector, es necesario llevar a cabo una serie de acciones que permitan mantenerla y fortalecerla en el mediano y largo plazo por medio del diseño e implementación de diversas estratégicas ajustadas al contexto actual de la dinámica económica y social (Sölvell, 2015). En este orden de ideas, las investigaciones sobre las estrategias planteadas inicialmente por Porter (1980) continua en desarrollo en función de mejorarlas y adaptarlas a las transformaciones que exigen los entornos de hoy día, destacando la evolución de las ideas originales del modelo hacia estrategias hibridas, permitiendo a las organizaciones la generación de múltiples fuentes de ventajas competitivas frente a la competencia, combinando factores relacionados, por ejemplo, con el liderazgo en costos y la diferenciación (Salavou, 2015); La tabla 1 expone una revisión y actualización del enfoque de las estrategias genéricas de Porter (1980).

Tabla 1: Evolución del enfoque de las estrategias del modelo de Porter (1980) (Adaptada de Salavou, 2015)

\begin{tabular}{|l|l|l|}
\hline \multicolumn{1}{|c|}{$\begin{array}{c}\text { Enfoque de las estrategias } \\
\text { originales del modelo de Porter }\end{array}$} & $\begin{array}{c}\text { Enfoque revisado y actualizado de } \\
\text { las estrategias }\end{array}$ & \multicolumn{1}{|c|}{ Aporte } \\
\hline $\begin{array}{l}\text { Crea opciones estratégicas } \\
\text { basadas en la teoría }\end{array}$ & $\begin{array}{l}\text { Crea opciones estratégicas } \\
\text { basadas en hechos }\end{array}$ & $\begin{array}{l}\text { La era de los datos e } \\
\text { información provee } \\
\text { suficiente fundamento para } \\
\text { la toma de decisiones en } \\
\text { este aspecto. }\end{array}$ \\
\hline $\begin{array}{l}\text { Ofrece un modelo que comprende } \\
\text { estrategias puras }\end{array}$ & $\begin{array}{l}\text { Sugiere muchas estrategias } \\
\text { híbridas a nivel empresarial }\end{array}$ & $\begin{array}{l}\text { La dinámica actual exige } \\
\text { una visión holística e } \\
\text { integral de las estrategias. }\end{array}$ \\
\hline $\begin{array}{l}\text { Ofrece opciones estratégicas en } \\
\text { dicotómicas }\end{array}$ & $\begin{array}{l}\text { Ofrece opciones estratégicas en } \\
\text { flexibles }\end{array}$ & $\begin{array}{l}\text { Las estrategias en el } \\
\text { contexto actual requieren } \\
\text { de mayor adaptabilidad al } \\
\text { cambio. }\end{array}$ \\
\hline Se ajusta al análisis sectorial & $\begin{array}{l}\text { Se ajusta al análisis sectorial o } \\
\text { multisectorial }\end{array}$ & $\begin{array}{l}\text { La interacción entre } \\
\text { diversos sectores, es } \\
\text { fundamental para diseñar y } \\
\text { ajustar las estrategias. }\end{array}$ \\
\hline
\end{tabular}

Tomando como referencia estos fundamentos teóricos sobre las ventajas competitivas es importante hacer algunas precisiones sobre la aplicabilidad de esta teoría en función del propósito del estudio con base en dos argumentos esenciales. En primer lugar, se destaca que Porter (2015) proporciona un marco referencial importante para la generación de ventajas competitivas que puedan ser aplicables a las pymes para la mejora de su posicionamiento en el sector, en tal sentido, el enfoque revisado de estas estrategias genéricas proporciona mayor flexibilidad y amplitud atendiendo a los cambios de paradigma que se están gestando actualmente en le dinámica económica y social (Carvalho y Costa, 2014). En segundo lugar, el aprovechamiento, integración y utilización de los recursos disponibles en la organización debe responder necesariamente a estos nuevos desafíos; por ello, es relevante que las técnicas y mecanismos para alcanzar la competitividad tengan como base la gestión adecuada y eficiente de factores críticos como la información, las mejores prácticas en temas de Tecnologías de la Información y Comunicación (TIC), el capital humano, gestión estratégica e innovación (Ahmedova, 2015; Ceptureanu, 2015).

\section{Gestión por procesos}

Existe evidencia en la literatura sobre la creciente importancia que tiene la implementación de la gestión por procesos en las organizaciones y el interés que esta cuenta por parte de los profesionales en los últimos años, a pesar de las limitaciones y el escaso desarrollo que se presenta en Hispanoamérica (Sánchez y Blanco, 2014). En este orden de ideas, Wolf y Harmon (2012) destacaron que efectivamente se evidencia un claro 
interés por implementar proyectos relacionados con la gestión de procesos, el cual no disminuyó a pesar de los períodos económicos difíciles por los que atraviesa la sociedad, comprendiendo la importancia que este tipo de iniciativas tiene para la mejora y el desarrollo de la organización; por otra parte, los autores señalan que ha habido un desarrollo geográfico importante principalmente hacia Europa y Estados Unidos, en donde la gestión por procesos ha pasado de ser una práctica informal a formar parte de las operaciones continuas de las empresas (Wolf y Harmon, 2012).

En este contexto, es necesario desarrollar algunas bases teóricas sobre lo que comprende la gestión por procesos y cómo contribuye a la mejora de la organización. Para comenzar, se debe mencionar que, a pesar de la diversidad de enfoques y definiciones que existe sobre el término, de acuerdo a la visión de algunos autores un proceso es un conjunto de actividades y procedimientos que interactúan de forma sinérgica, lógica y secuencialmente para transformar unos insumos (inputs) en una salida (outputs) para crear valor y satisfacer una necesidad puntual del entorno (Davenport y Short, 1990; Simon, 2018). Ante este planteamiento, es relevante precisar que los procesos cuentan entonces con una serie de características comunes que lo definen, a saber: a) Cuentan con una entrada específica, es decir, un insumo el cual debe ser transformado y que proviene de un proveedor interno o externo, b) Involucra personas con diferentes niveles de responsabilidad y liderazgo en cada uno de sus componentes; c) Requiere de recursos materiales e intelectuales para su desarrollo; d) Implica una fase de planificación para determinar su objetivo y detalle a nivel de procedimientos; e) Termina cuando hay una salida o producto de acuerdo a las características y requerimientos establecidos (Rosemann y Vom Brocke, 2015).

Con base en los argumentos teóricos señalados, se puede entender de forma concreta que gestión por procesos en la organización consiste en identificar objetivos para diseñar y desarrollar acciones que integren un conjunto de medidas de control, administrativas y de supervisión con el fin de orientar las actividades hacia los objetivos y metas organizacionales, siempre tomando en cuenta las necesidades de los clientes y alineados con sus expectativas (Chang, 2016). De forma ilustrativa, la figura 1 expone la interrelación de los elementos que conforman una visión general de los procesos en la organización.

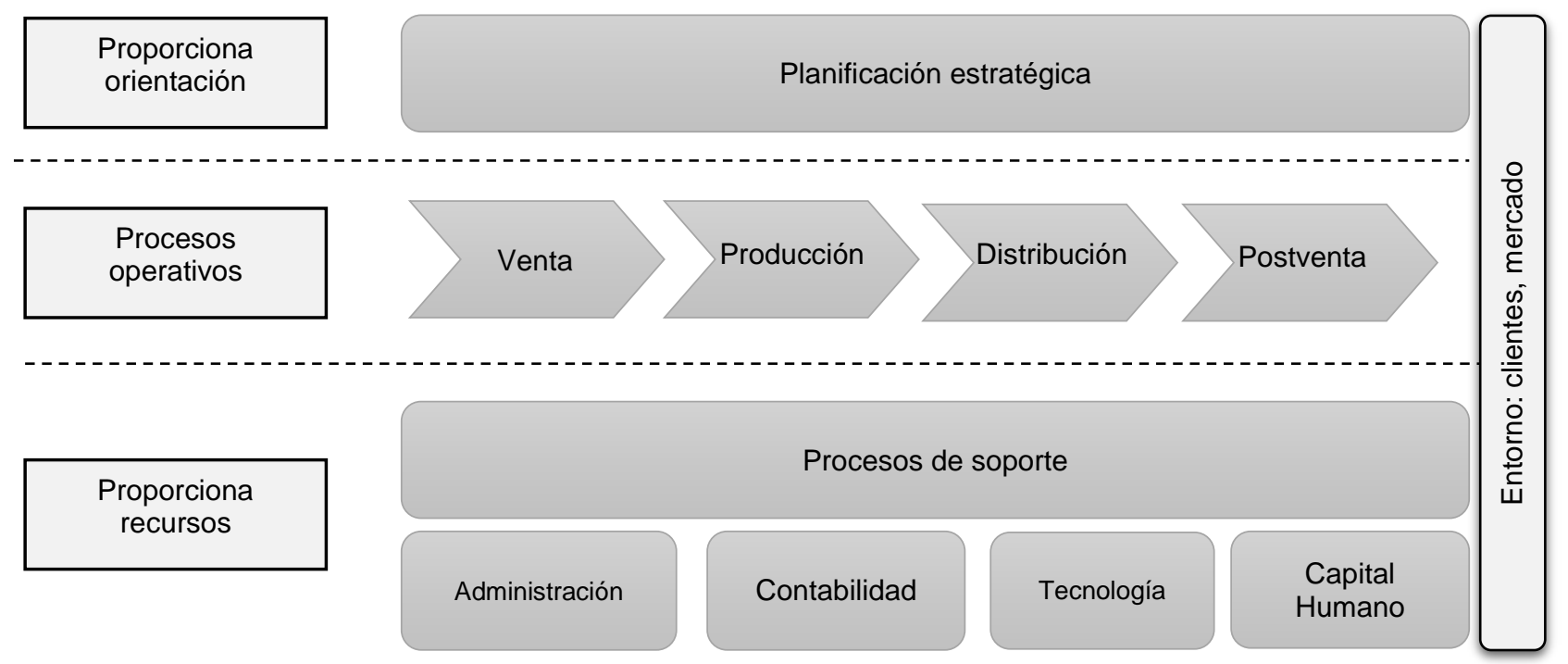

Fig. 1: Procesos en la organización (Adaptada de Mallar, 2010)

De acuerdo a los elementos presentados en la figura 1, la gestión por procesos integra diversas áreas de la organización, la interacción entre estas se transforma en un factor clave para alcanzar la integración de las actividades y procedimientos. Su relación con la competitividad radica en que es posible alcanzarla por medio de la medición de los resultados utilizando para ello indicadores; el compromiso y capacitación adecuada del recurso humano que permita una apropiación de la gestión por procesos en la cultura organizacional; la alineación con las metas y objetivos estratégicos del negocio; por último, la tecnología como mecanismo de apoyo para llevar a cabo todas las actividades y procedimientos necesarios en la organización (Vuksic, et al., 2017).

\section{METODOLOGÍA}

Para la realización de este artículo se planteó un tipo de investigación cualitativa. Se optó por este enfoque tomando en consideración aspectos reseñados por autores como Talaya y Collado (2014), quienes han propuesto esta alternativa de análisis y abordaje, por facilitar un acercamiento a la realidad de fenómenos y eventos que pueden ser contrastados con referentes teóricos, permitiendo a los investigadores utilizar la 
experiencia previa que se haya desarrollado como parte de su proceso o contacto con los eventos de estudio. Asimismo, la revisión documental ha servido como base para la exploración de las teorías sobre un tema particular para la construcción de conocimiento (Wolfswinkel, 2013). Las fuentes secundarias consultadas en su mayoría fueron artículos científicos y documentos de bases de datos como Scielo, Scopus, Elseiver y Emerald siquiendo con las pautas y criterios establecidos universalmente, es decir, de preferencia de los últimos 5 años en revistas de corriente principal y mayor porcentaje en idioma inglés. Así también, se tomaron en consideración publicaciones de fuentes gubernamentales y no gubernamentales para lograr la recopilación de interés al respecto y nutrir la información que se recopiló para posteriormente destacar los aspectos de mayor importancia. Como técnica para la captura de la información se desarrolló una revisión documental, cuyas etapas se muestran seguidamente, en la figura 2.

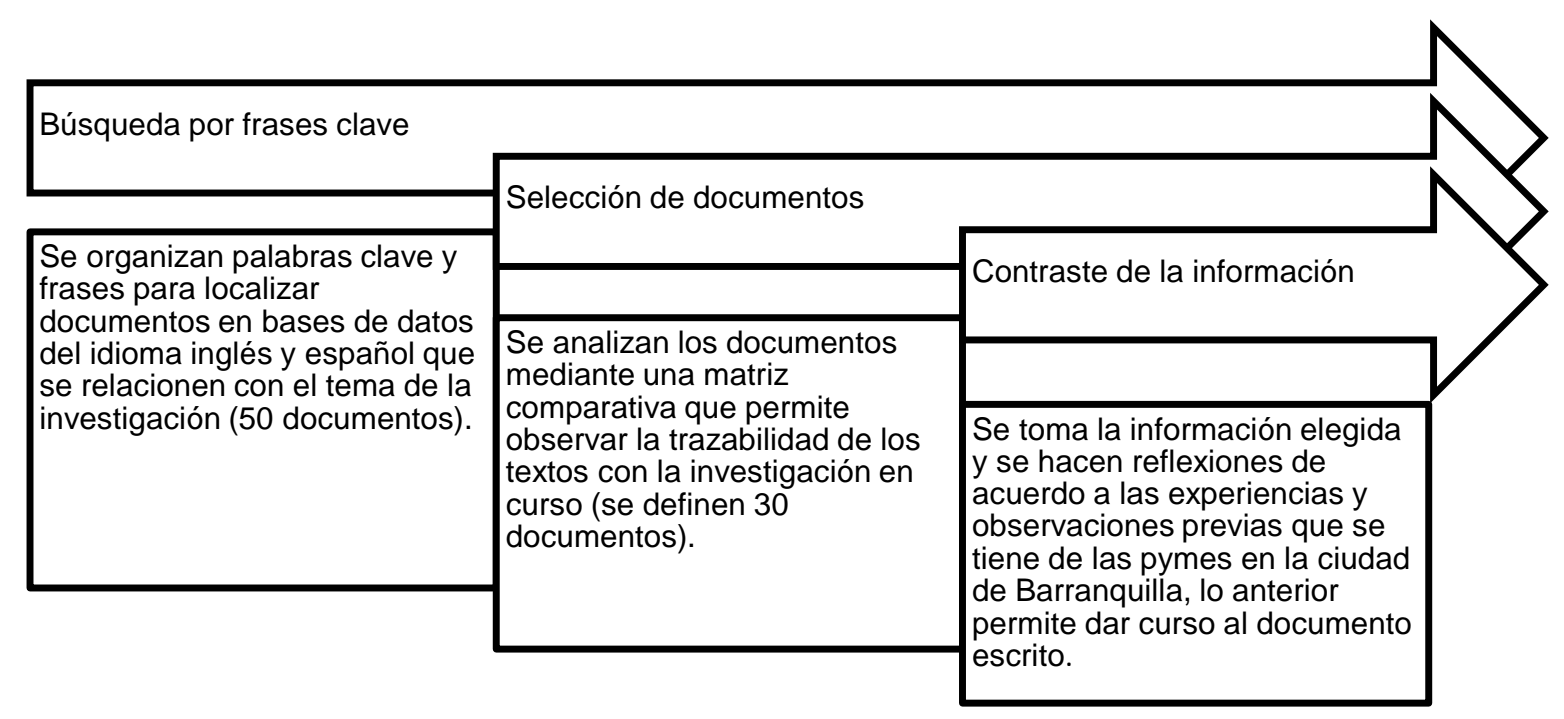

Fig. 2: Etapas de la revisión documental

Bajo el enfoque metodológico establecido, la revisión de la literatura siguió unas pautas muy bien definidas que facilitaran la organización y sistematización de la información. En primer lugar se definieron tres ejes temáticos que permitieran orientar la búsqueda y recolección de documentos por medio de la utilización de un conjunto de palabras clave, en este sentido, la primera línea de trabajo fue con respecto a la posición actual en el ámbito de competitividad que tienen las pymes colombianas frente a sus pares a nivel internacional, para ello se utilizaron palabras clave en español e inglés que permitieran hallar documentos oficiales reconocidos internacionalmente sobre los avances que, en materia de competitividad, Colombia ha tenido. Se emplearon las siguientes frases: índice de competitividad Colombia, Banco Mundial, Competitividad Colombia, Foro Económico Mundial, innovación, pymes, desarrollo, para de este modo lograr siete criterios de búsqueda de información.

Asimismo, el siguiente grupo de palabras clave se establecieron en función de un segundo eje temático, las herramientas para alcanzar la competitividad. En tal sentido, se emplearon frases como: herramientas empresariales, competitividad, calidad, procesos, gestión de la calidad, cadena de valor, análisis de procesos, reingeniería de procesos, gestión por procesos, indicadores y fichas de procesos, factores determinantes de competitividad, procesos administrativos, pyme, indicadores, para un total de quince criterios. Por último, se analizaron las características económicas de la región caribe aplicando las siguientes líneas de búsqueda: contribución al PIB, sectores económicos más relevantes, emprendimiento, condiciones medioambientales que favorecen su desarrollo, emprendimiento e inversión, contribución de la pyme a la economía y el empleo, factores de competitividad de la pyme y estrategias para la sostenibilidad de la pyme.

En segundo lugar, una vez obtenida todos los artículos e informes recolectados con base en los criterios de búsqueda establecidos se procedió a la realización de un proceso de selección en el cual se incluyeran los documentos más relevantes en función del objetivo del estudio. Para ello, se analizaron los aportes de cada autor, señalados en el resumen, sección de resultados, discusiones y conclusiones para el caso de artículos científicos y datos y estadísticas relevantes emanados de los organismos oficiales reconocidos internacionalmente sobre temas de competitividad, de este modo, se logró la depuración de la información para obtener finalmente treinta documentos de trabajo. Finalmente, en la tercera etapa se contrastaron los aportes y reflexiones de la literatura con base en las experiencias y observaciones que se tienen de la pyme en la Ciudad de Barranquilla con la finalidad de establecer un marco de comparación que diera cuenta de su situación actual en términos de competitividad y cómo la gestión por procesos puede contribuir a mejorar esta 
posición. Los ejes que orientaron el análisis fueron los establecidos inicialmente, posición actual en el ámbito de competitividad que tienen las pymes colombianas frente al a otras a nivel internacional, herramientas para lograr la competitividad y el contexto económico de la pyme en la región caribe colombiana.

\section{RESULTADOS}

El concepto de competitividad ha tomado tal relevancia, que no solo se asocia al entorno empresarial, sino también al avance económico de los países y entes territoriales, como el Sistema Nacional de Competitividad de Colombia formado en 2005 con la participación de Michael Porter, el cual se encarga de promover y poner en marchar políticas de productividad y competitividad a nivel nacional, con base en el Informe Nacional de Competitividad, con datos de las áreas de mayor impacto como salud, ciencia, tecnología, mercado laboral, financiación para el emprendimiento, entre otras (SNCCTI, 2018). A nivel internacional, se encuentran iniciativas promovidas por el Foro Económico Mundial y el Banco Mundial, entre otros (Hoyos y Salazar, 2011). Todas estas, guardan en común los lineamientos que dan forma o cavidad a la competitividad en la empresa de hoy, y que indistintamente del renglón productivo donde se ubica una organización u otra se deben contemplar para alcanzar los niveles de excelencia y participación en los mercados que se desean obtener (Hernández et al., 2014). A continuación, en la tabla 2 se expone la información en donde Colombia figura en términos de competitividad frente a otros países.

Tabla 2: Indicadores de Competitividad 2017-2018 (Datos tomados de SNCCTI, 2018)

\begin{tabular}{|l|l|c|c|l|}
\hline \multicolumn{1}{|c|}{ Nombre } & \multicolumn{1}{|c|}{ Organización } & No. países & Posición & \multicolumn{1}{c|}{ Top 5 } \\
\hline $\begin{array}{l}\text { Índice de } \\
\text { Competitividad Global }\end{array}$ & Foro Económico Mundial & 137 & 66 & $\begin{array}{l}\text { Suiza, Estados Unidos, } \\
\text { Singapur, Países Bajos y } \\
\text { Alemania }\end{array}$ \\
\hline $\begin{array}{l}\text { Anuario Mundial de } \\
\text { Competitividad }\end{array}$ & $\begin{array}{l}\text { Instituto Internacional para } \\
\text { el Desarrollo Gerencial }\end{array}$ & 63 & 54 & $\begin{array}{l}\text { Hong Kong, Suiza, Singapur, } \\
\text { Estados Unidos y Países Bajos }\end{array}$ \\
\hline $\begin{array}{l}\text { Índice de Facilidad } \\
\text { para hacer Negocios }\end{array}$ & Grupo del Banco Mundial \\
\hline $\begin{array}{l}\text { Índice Global de } \\
\text { Innovación }\end{array}$ & $\begin{array}{l}\text { Organización Mundial de la } \\
\text { Propiedad Intelectual } \\
\text { (OMPI), la Universidad } \\
\text { Cornell y la Escuela de } \\
\text { Negocios INSEAD }\end{array}$ & 190 & 59 & $\begin{array}{l}\text { Nueva Zelanda, Singapur, } \\
\text { Dinamarca, la Republica de } \\
\text { Coreo y Hong Kong }\end{array}$ \\
\hline
\end{tabular}

De acuerdo a las cifras citadas, Colombia entre 2017 y 2018 se ha ubicado en algunos registros en la media de los países analizados, teniendo que el mejor desempeño se observa en el índice de Competitividad Global (ICG), donde de los 137 países que fueron revisados, Colombia logró la posición 66. Caso similar en el Índice de Facilidad para hacer Negocios (IFHN) del Grupo Banco Mundial donde de 190 países se instaló en el puesto 59. No obstante, en el caso del Instituto Internacional para el Desarrollo Gerencial (INDG) se muestra en la casilla 54 de 63 países examinados (SNCCTI, 2018). El Foro Económico Mundial durante cuatro décadas mediante sus reportes anuales, ha estudiado y evaluado los factores que sustentan la competitividad de las naciones, con el objeto de hacer visible y estimular la discusión entre todos los interesados, tanto privados como gobierno, sobre las estrategias y políticas para enfrentar los retos de las mejoras en competitividad (Smit, 2010).

Las herramientas empresariales para lograr posiciones competitivas son variadas, además del análisis de la cadena de valor se tienen otras propuestas como el análisis de procesos, reingeniería de procesos, gestión de calidad, entre otros, tomando mucha relevancia en los últimos años la gestión por procesos. Esto último se puede entender por la madurez que han alcanzado los procesos administrativos y porque a hoy se cuenta con mucha información que puede ser de utilidad, incluyendo los primeros anticipos logrados en el siglo anterior (Hammer, 2015). Entre finales de los 90 e inicios del año 2000, la gestión por procesos ha sido ampliamente usada por empresas con base en gestión de calidad o calidad total, al centrar su enfoque en la identificación y gestión de los procesos y la interacción que se da entre ellos, apuntando al diseño de procesos pensando en los clientes, contribuyendo de forma sostenida a conseguir resultados. Esta gestión por procesos busca que las estructuras empresariales tengan una mayor capacidad de adaptación y respuesta a los constantes cambios del mercado, con un enfoque mayor hacia el cumplimiento de los objetivos, el valor agregado y la satisfacción del cliente (Ruiz et al., 2014).

Para su gestión, se parte de identificar en los procesos, definir la relación entre ellos a través de un mapa de procesos, describir las actividades a través de diagramas, soportar la información en una ficha de proceso 
donde entre otros temas, se establecen indicadores que permitirán medir la capacidad, eficiencia y efectividad de los procesos. La información de los indicadores permitirá hacer un análisis de la operación, detectar oportunidades de mejora y tomar decisiones (Ruiz et al., 2014). Las pequeñas y medianas empresas (pymes) pueden encontrar panoramas diferenciales, donde no solo logren dominar los aspectos básicos de su organización, sino generar una dinámica estratégica que les permita anticipar y hacer frente a los cambios del mercado. Los aspectos esenciales citados, se compilan seguidamente en la figura 3. Como se observa, la gestión por procesos debe cumplir un ciclo particular, donde cada organización demarque sus necesidades, peculiaridades y establezca así también sus mecanismos o instrumentos de gestión, mejora y seguimiento.

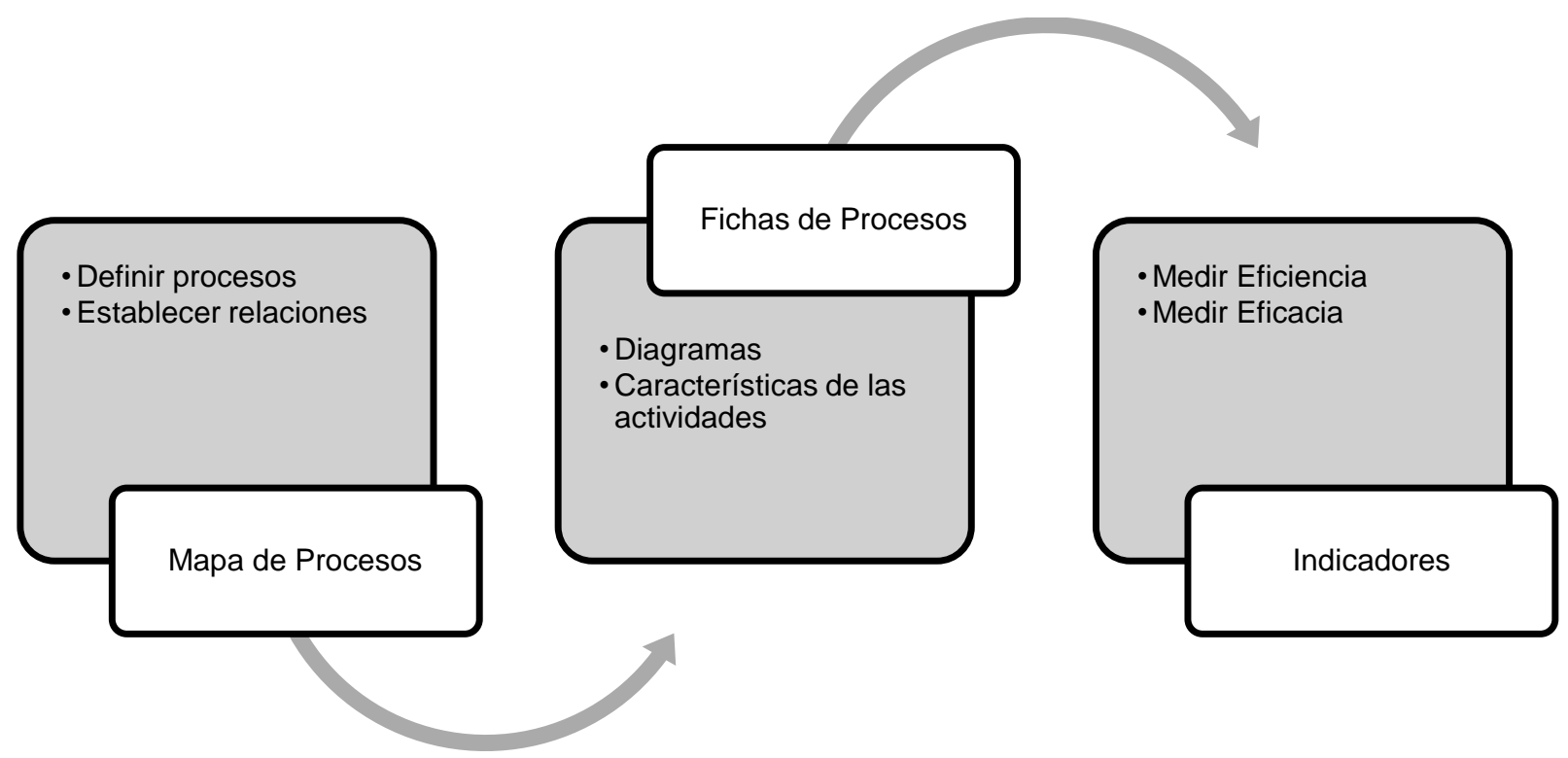

Fig. 3: Herramientas para la gestión por procesos a nivel de pymes

La región caribe colombiana es uno de los sectores de mayor importancia para la economía del país, ubicándose en la última década como una de las regiones con mayor contribución al PIB nacional con datos de 2013, debido principalmente al impulso minero y el crecimiento del sector construcción. Las condiciones naturales de la región por presencia de recursos naturales y su ubicación geográfica, hacen de esta zona del país un territorio propicio para procesos de emprendimiento, lo cual se refleja en las cifras del año 2014 donde tuvo las tasas más significativas en materia de emprendimiento, por encima de otras ciudades en el país como Medellín, Bogotá y Bucaramanga. La región en los últimos años se ha convertido en un lugar atractivo para nuevas inversiones de nuevos negocios o expansión, con cifras de inversión de alrededor US\$30.000 y generación de 17.000 puestos de empleo entre 2010 y 2015 (Cardona et al., 2017).

Este dinamismo en las cifras de emprendimiento aplica para las pymes, en las que se convierten posteriormente estas nuevas unidades, y tener las herramientas adecuadas para garantizar su sostenibilidad en el tiempo y participación en el mercado pasan a ser sus principales factores de éxito; autores como Bansal y Des Jardine (2014), han dejado ver en sus investigaciones que la sostenibilidad ya no es un tema trivial y que por el contrario hoy debe estar en la agenda de trabajo de cualquier tipo de organización empresarial. En Colombia las pymes representan el $96.4 \%$ de total de empresas, según datos del Departamento Administrativo Nacional de Estadística (DANE) de 2005 y generan el 63\% del empleo del país, representando una base importante para el comportamiento del PIB nacional, aportando así al desarrollo de la economía, productividad y competitividad (Castellanos et al., 2016). A pesar de esta relevancia de las pymes, se presenta un gran porcentaje de ellas que no logra mantenerse a flote en los primeros años siendo un aspecto crítico en esto la falta de competitividad (Castellanos et al., 2016).

Todo lo enunciado toma importancia, pues los conceptos antes revisados de competitividad y las herramientas disponibles para alcanzarla, rescatando la posibilidad del uso de la gestión de procesos, a partir de los comentarios expuestos, resaltan el creciente uso de esta metodología en los últimos años, logrando flexibilizar a las empresas de manera dinámica en sus estrategias, para hacer frente a los cada vez más vertiginosos y exigentes entornos empresariales (Balkyte y Tvaronavičiene, 2010). Los mercados actuales están influenciados por un gran intercambio de información gracias a la globalización y redes sociales, donde los clientes y usuarios de servicios de empresas grandes y pequeñas tienen acceso a conocer de nuevos productos, avances en tecnología, mecanismos tecnológicos, entre otros, que promueven una mayor demanda en especificaciones volubles para sus necesidades en comparación con años atrás. La velocidad de respuesta de las pymes tiene que ser mayor para poder asegurar la sostenibilidad de su posición en el mercado (Tello y Velasco, 2016). 
Los factores de competitividad mencionados por García et al. (2014), en su análisis, dan una idea de los aspectos que debe tener en cuenta una pyme, como son: innovación, capital humano, tecnologías de información y comunicación, medio ambiente, recursos financieros y factores de diferenciación. La gestión por procesos aplica los sistemas de calidad y gestión de operaciones para lograr eficiencia y eficacia organizacional, impulsando acciones de mejora con base en la información generada del seguimiento y control, lograda a través del seguimiento de indicadores de los aspectos clave de los procesos. En ésta se busca un enfoque hacia los requerimientos de los clientes internos y externos, asegurando un enfoque homologado en todas las áreas (Ramírez et al., 2016).

Según el análisis de Sánchez et al. (2011), los motivos que impulsan a las empresas a seguir este modelo de gestión por procesos son: mejora en eficiencia y productividad, logro de objetivos corporativos, mejora en la imagen corporativa, mantenimiento o incremento en la participación del mercado y la obtención de ventajas competitivas. Por consiguiente, una organización que adopta una gestión por procesos, podría diferenciarse de manera palpable de otra que no aplica este tipo de lineamientos para sus actividades organizacionales, entre otras cosas porque se estimulan aspectos como creatividad e innovación (Palma et al., 2016). Así mismo destacan los mencionados investigadores que el sistema presenta algunas limitaciones para su implementación como la resistencia al cambio, estrategia defectuosa, apego a antiguas tecnologías, baja inversión en educación, equipo directivo inflexible, falta de compromiso a largo plazo y falta de tiempo para planificar por parte de la dirección. Sin embargo, no ha sido motivo para evitar que cada vez más empresas acojan este sistema, por sus reconocidos beneficios: conocimiento del por qué se hacen las cosas al identificar cada proceso, esto a su vez permite identificar los costos asociados y diseñar estrategias para su reducción; contra con un sistema de medición; favorecimiento del trabajo en equipo; orientación hacia el cliente; identificación de obstáculos; disminución de errores; permite el análisis del valor agregado asociado a cada actividad; aporta una visión global de la organización; ayuda a la toma de decisiones; favorece la implementación de sistemas de calidad total y mejoramiento continuo; mejor uso de recursos y elimina las barreras entre los diferentes departamentos de la organización (Hernández et al., 2017).

Por las múltiples ventajas ya mencionadas, la gestión por procesos tiene mucho que aportar a la gestión de las pymes a nivel mundial, al ser una herramienta que facilita la mejora continua, permite detectar oportunidades de mejora de manera ágil y orienta a la compañía en un objetivo único de satisfacción al cliente y al tener identificadas las posibles limitaciones para su aplicación, solo resta definir una metodología adecuada para cada organización, que tenga en cuenta sus particularidades y las del sector en que se desenvuelva, para que sea un éxito su evolución y sostenibilidad y por ende, lleve a obtener los resultados de competitividad esperados (Durst y Runar, 2012). Sánchez et al., (2011), sugirieron una metodología para ser aplicada en pymes españolas, que también podría ser considerada para pymes en Colombia y la región. Dicha metodología propone la implementación de 5 etapas: i) equipo humano comprometido; ii) diagnóstico inicial; iii) planificación; iv) formación del personal; y v) identificación de los procesos. La existencia de un equipo comprometido y con un alto sentido de pertenencia; la realización de diagnóstico inicial; el cumplimiento de la etapa de planificación del proyecto; la formación y sensibilización del personal de forma constante; la identificación de los procesos de la empresa, para su clasificación y análisis; los procedimientos de control (establecimiento de indicadores), puede dar como resultado final la mejora de procesos en los entes económicos (Schmiedel et al., 2014).

\section{DISCUSIÓN FINAL}

Siendo las pymes un modelo empresarial de mucha importancia en Colombia y con un impacto tan interesante como el analizado en el presente documento, se hace recomendable la motivación para aplicar el sistema de gestión por procesos con miras a promover un aporte mejor a la economía local, nacional y mundial; de esta manera se pueden alcanzar niveles de gestión diferenciadores, que no solo promuevan la competitividad como lo ha expresado Porter (2015), en beneficio de los empresarios y sus colaboradores, sino que se logre la dinamización misma de los mercados en la medida que los niveles organizacionales alcancen escalas más estructuradas. Por otro lado, la gestión por procesos deriva también en mayor conocimiento del ente productivo lo cual puede verse traducido en términos de sostenibilidad, tal como lo ha planteado Bansal y Des Jardine (2014), indicando que toda organización debe incluir hoy en su agenda de trabajo, las estrategias necesarias que apunten a esta posición.

Con base en los resultados es posible argumentar que la evidencia mostrada por Wolf y Harmon (2012) es acertada en cuanto al escaso desarrollo que tiene la gestión de procesos en países hispanoamericanos y contrasta con los índices de competitividad que actualmente posee el país, donde la pyme desempeña un rol fundamental (SNCCTI, 2018). Por otra parte, las características y el contexto particular que tienen estas organizaciones en la dinámica económica de la región caribe, permiten reflexionar sobre la pertinencia de la implementación de la gestión por procesos, la cual señalan Vuksic, et al. (2017) como un mecanismo efectivo para lograr la competitividad. Otro factor sobre el cual se puede plantear la discusión es si la gestión por 
procesos implica un cambio profundo en la cultura organizacional, en este sentido, Rosemann y Vom Brocke (2015) señalan que el factor humano en la implementación de esta dinámica es crucial para lograr el éxito de esta práctica administrativa. Por otra parte, el aporte que puede dar la gestión por proceso a la pyme de la ciudad de Barranquilla es notorio, dado que esta práctica podría contribuir a superar con éxito los primeros años de vida de una empresa a través del impulso de su competitividad, considerado que esto es una debilidad generalizada, tal como lo expone Castellanos et al. (2016).

Por lo manifestado, la búsqueda de la competitividad como motor empresarial que brinda a toda corporación la posibilidad de ocupar una posición destacable en un mercado de constante cambio, puede propiciar al interior de toda empresa, la transformación necesaria para beneficiar de forma simultánea su participación en el entorno y la gestión por procesos como un factor clave y particular en su escenario de acción, lo anterior se alinea con postulados propuestos por investigadores contemporáneos como Sánchez et al. (2016), quienes en sus estudios han logrado recopilar evidencias de la influencia de la organización estructurada en la competitividad global de toda célula empresarial y que muestran como los esfuerzos constantes y determinados en tal sentido pueden arrojar resultados no esperados.

\section{CONCLUSIONES}

De acuerdo al trabajo presentado y a los resultados obtenidos, se pueden plantear las siguientes conclusiones principales:

a) La evidencia mostrada en el artículo permite contribuir al desarrollo de un marco general para el diseño de políticas orientadas a la implementación de la gestión por procesos en las pymes de la ciudad de Barranquilla, sustentado en la relevancia, pertinencia e interés que tiene este campo en la actualidad. En tal sentido, se debe fomentar el compromiso de los sectores involucrados, sean de orden público o privado, para que logren actuar coordinadamente en la aplicación de estos mecanismos de mejora en las organizaciones; asimismo, con la participación responsable de los líderes y todo personal de la pyme;

b) Los datos y estadísticas sugeridos por la literatura sobre la dinámica económica, innovación y emprendimiento de la región caribe, muestran que hay un auge económico importante y, por tanto, es posible aprovechar los recursos de la pyme para impulsar su competitividad; por tal razón, existe una clara oportunidad para canalizar a estas organizaciones hacia estados más prósperos. Ante este planteamiento, es pertinente mencionar que el contexto actual sirve como referencia para la implementación de gestión por procesos en otras experiencias similares de países emergentes como el caso colombiano, dado que las características, circunstancias y factores de éxito de las pymes son parecidas;

c) La literatura confirma que existe un impacto en la competitividad si se adoptan medidas por implementar la gestión por procesos en las organizaciones. Tal evidencia sustenta el argumento principal de la investigación y coloca a los investigadores en un rol más activo que les permita indagar y comprender con mayor profundidad los factores multidimensionales que debe atender la pyme para lograr niveles de competitividad adecuados a los niveles internacionales; aún más conociendo que este sector es el más importante y representativo de la economía nacional.

\section{REFERENCIAS}

Ahmedova, S., Factors for Increasing the Competitiveness of Small and Medium-sized Enterprises (SMEs) in Bulgaria, Procedia-Social and Behavioral Sciences, 195, 1104-1112 (2015)

Balkyte, A. y M. Tvaronavičiene, Perception of Competitiveness in the Context of Sustainable Development: Facets of "Sustainable Competitiveness", Journal of Business Economics and Management, 11(2), 341-365 (2010)

Bansal, P. y M. Des Jardine, Business Sustainability: It is About Time, Strategic Organization, 12(1), 70-78 (2014)

Benítez, M., Evolución del Concepto de Competitividad, Ingeniería Industrial, Actualidad y Nuevas Tendencias, 3(8), 75$82(2012)$

Cardona, D., A. Rada y H. Palma, Creación de Empresa como Pilar para el Desarrollo Social e Integral de la Región Caribe: Apuntes críticos, Saber, ciencia y libertad, 12(1), 15 (2017)

Carvalho, L. y T. Costa, Small and Medium Enterprises (SMEs) and Competitiveness: An Empirical Study, Journal of Management Studies, 2(2), 88-95, (2014)

Castellanos, L.E.F., K. Insignares y L. Vega, La Innovación como Factor Clave para Mejorar la Competitividad de las pymes en el Departamento del Atlántico-Colombia, Dictamen Libre, (16), 21-36 (2016)

Ceptureanu, S. I., Competitiveness of SMES, Business Excellence and Management Review, 5(2), 55-67 (2015) 
Chang, J.F., Business process management systems: strategy and implementation, Auerbach Publications (2016)

Confederación Nacional de Cámaras de Comercio (Confecámaras), Informe de Dinámica Empresarial en Colombia en 2016, Universidad Santo Tomas, Bucaramanga, Colombia (2016)

Consuegra, I., K. Barrios, J. Contreras y E. Acosta, Unidad de Apoyo al Microempresario: Hacia la construcción de Microempresas sostenibles, Barranquilla, Universidad Simón Bolívar, Colombia (2017)

Davenport, T.H. y Short, J. E., The New Industrial Engineering: Information Technology and Business Process Redesign, Sloan Management Review, 31(4), 11 (1990)

Durst, S.E, e I. Runar, Knowledge Management in SMEs: A Literature Review, Journal of Knowledge Management, 16(6), 879-903 (2012)

García, J.H., R. Prieto y D. Sanz, Factores de Competitividad Organizacional. Su Gestión para la Sostenibilidad Empresarial, VI jornadas científicas nacionales Dr. José Gregorio Hernández, Maracaibo, Venezuela, Noviembre (2014)

Graham, A., Airport Strategies to Gain Competitive Advantage, In Airport Competition, 109-122, Routledge (2016)

Gupta, S., N. Malhotra, M. Czinkota y P. Foroudi, Marketing innovation: A consequence of competitiveness, Journal of Business Research, 69(12), 5671-5681 (2016)

Hammer, M., What is Business Process Management, Handbook on Business Process Management, 1, 3-16 (2015)

Hernández, A., C. Marulanda y M. López, Análisis de Capacidades de Gestión del Conocimiento para la Competitividad de Pymes en Colombia, Información Tecnológica, 25(2), 111-122 (2014)

Hernández, H.G., D. Cardona y J. Del Rio, Direccionamiento Estratégico: Proyección de la Innovación Tecnológica y Gestión Administrativa en las Pequeñas Empresas, Información Tecnológica, 28(5), 15-22 (2017)

Hoyos, A.T. y G. Salazar, Análisis de Capacidades y Evolución del Sistema Nacional de Ciencia, Tecnología e Innovación en Colombia, Cuadernos Latinoamericanos de Administración, 7(12), 49-60 (2011)

Mallar, M.Á., La Gestión por Procesos: Un Enfoque de Gestión Eficiente, Visión de Futuro, 13(1), (2010)

Nechaev, A.S., O. Antipina y A. Prokopyeva, The Risks of Innovation Activities in Enterprises, Life Science Journal, 11(11), 574-575 (2014)

Palma, H.G., D. Arbeláez y A. Pardo, La Innovación Empresarial como Impulsora de las Pymes en la Región Caribe, Revista Panorama Económico, (24), 9 (2016)

Porter, M. y J. Heppelmann, How Smart, Connected Products Are Transforming Companies, Harvard Business Review, 93(10), 96-114 (2015)

Porter, M.E. y M. Kramer, Creating Shared Value, In Managing Sustainable Business, DOI: 10.1007/978-94-024-1144-7, Springer, 327-350 (2018)

Porter, M.E., Competitive Strategy, Techniques for Analyzing Industries and Competitors, Free Press, New York, NY (1980)

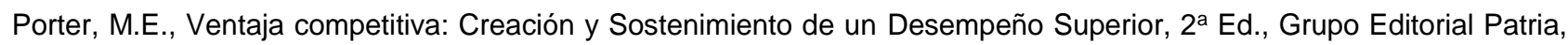
Ciudad de México, México (2015)

Ramírez, L.S.M., J. Beltrán y E. Nieto, Gestión por Procesos como Factor de Competitividad de Pymes del Sector Industrial en el Estado de Querétaro, Red Internacional de Investigadores en Competitividad, 9(1), 816-832 (2016)

Rosemann, M. y J. Vom Brocke, The six core elements of business process management, In Handbook on business process management, 1, 105-122, Springer, Berlin, Heidelberg (2015)

Ruiz-Fuentes, D., R. Almaguer-Torres, I. Torres-Torres y A. Hernández-Peña, La Gestión por Procesos, su Surgimiento y Aspectos Teóricos, Ciencias Holguín, 20(1), 10-19 (2014)

Sánchez, L. y B. Blanco, La Gestión por Procesos. Un campo por Explorar, Dirección y Organización, (54), 54-71 (2014)

Sanchez-Gutierrez, J., J. Mejía-Trejo, J. Vargas-Barraza y G. Vazquez-Avila, Intellectual Capital, Impact Factor on Competitiveness: Manufacturing Industry SMEs in Mexico, Measuring Business Excellence, 20(1), 1-11 (2016)

Sánchez-Ruiz, L., B. Blanco, C. Pérez-Labajos y A. Serrano, La Gestión por Procesos: Una Herramienta para Lograr la Supervivencia de las Pymes, Cajamarca, Colombia (2011)

Schmiedel, T., J. Vom-Brocke y J. Recker, Development and Validation of an Instrument to Measure Organizational Cultures Support of Business Process Management, Information and Management, 51(1), 43-56 (2014)

Simon, H.A., The Proverbs of Administration, In Democracy Bureaucracy and the Study of Administration, 38-59, Routledge (2018)

Sistema Nacional de Competitividad, Ciencia, Tecnología e Innovación (SNCCTI), Indicadores Nacionales e Internacionales, Colombia (2018)

Smit, A.J., The Competitive Advantage of Nations: is Porter's Diamond Framework a New Theory that Explains the International Competitiveness of Countries? Southern African Business Review, 14(1) (2010) 
Sölvell, Ö., The Competitive Advantage of Nations 25 years-opening up new perspectives on competitiveness, Competitiveness Review, 25(5), 471-481 (2015)

Talaya, Á.E. y A. Collado, Investigación de Mercados, $1^{\text {a }}$ Ed., 8-209, Esic Editorial, Madrid, España (2014)

Tello, E.A. y J. Velasco, Inteligencia de Negocios: Estrategia para el Desarrollo de Competitividad en Empresas de Base Tecnológica, Contaduría y Administración, 61(1), 127-158 (2016)

Vuksic, V. B., M.P. Bach y D.S. Vugec, Understanding the Soft Side of BPM as a Competitiveness Driver: a Preliminary Research, Theory and Applications in the Knowledge Economy, 161, (2017)

Wolf, C. y P. Harmon, The State of Business Process Management; BP Trends, USA, (2012)

Wolfswinkel, J.F., E. Furtmueller y C.P. Wilderom, Using Grounded Theory as a Method for Rigorously Reviewing Literature, European Journal of Information Systems, 22(1), 45-55 (2013)

Yusuf, Y.Y., A. Gunasekaran y otros cuatro autores, A Relational Study of Supply Chain Agility, Competitiveness and Business Performance in the Oil and Gas Industry, International Journal of Production Economics, 147, 531-543 (2014) 
